\title{
Ataxia-telangiectasia with a novel ATM gene mutation and Burkitt leukemia: A case report
}

\author{
FANGHUA YE $^{1}$, WENWEN CHAI ${ }^{2}$, MINGHUA YANG $^{1}$, MIN XIE $^{1}$ and LIANGCHUN YANG ${ }^{1}$ \\ ${ }^{1}$ Department of Pediatrics, Xiangya Hospital, Central South University; ${ }^{2}$ Department of Nuclear Medicine, \\ Hunan Cancer Hospital and The Affiliated Cancer Hospital of Xiangya School of Medicine, \\ Central South University, Changsha, Hunan 410008, P.R. China
}

Received August 9, 2018; Accepted September 17, 2018

DOI: $10.3892 / \mathrm{mco} .2018 .1721$

\begin{abstract}
Ataxia-telangiectasia (A-T) is an infrequent autosomal recessive disorder that involves multiple systems and is characterized by progressive cerebellar ataxia, oculocutaneous telangiectasias, radiosensitivity, immune deficiency with recurrent respiratory infections, and a tendency to develop lymphoid malignancies. A-T is caused by mutations in the ATM gene, with $>1,000$ mutations reported to date and gradually increasing in number. Patients with A-T have an increased incidence of cancers. The aim of the present study was to retrospectively review the case of a patient who presented at the age of 5 years with cerebellar ataxia without telangiectasia, and was diagnosed with Burkitt leukemia by bone marrow biopsy and molecular testing at the age of 7 years at the Xiangya Hospital of Central South University (Changsha, China). The patient received chemotherapy with the pediatric CCCG-BNHL-2015 regimen (R4 group) and achieved a complete remission after 2 courses. However, recurrent respiratory infections and thrombosis occurred during chemotherapy. The diagnosis of A-T was confirmed by uncovering two variants of the ATM gene, including c.742C $>$ T (p.R248X; rs730881336) in exon 7 and c.6067-c.6068 ins GAGGGAAGAT in exon 41 by whole-exome sequencing. Unfortunately, the patient's parents refused follow-up treatment and he succumbed to recurrent severe infections 4 months after the diagnosis of Burkitt leukemia. The diagnosis of A-T may be challenging, as its phenotype can be incomplete early in the course of the disease. Detailed medical history, characteristic
\end{abstract}

Correspondence to: Dr Liangchun Yang, Department of Pediatrics, Xiangya Hospital, Central South University, 87 Xiangya Road, Changsha, Hunan 410008, P.R. China

E-mail: yangliangchung@163.com

Abbreviations: A-T, ataxia-telangiectasia; MTX, methotrexate; IT, intrathecal; CTX, cyclophosphamide; Ara-C, cytosine arabinoside; PARP, poly ADP-ribose polymerase; ATR, ATM-related

Key words: ataxia-telangiectasia, ATM gene, cancer susceptibility, immune deficiency, exome sequencing clinical manifestations and increasingly developed exome sequencing techniques may be helpful in diagnosing this rare disease. Management should be based on multidisciplinary guidance and other treatment options must be investigated in the future.

\section{Introduction}

Ataxia-telangiectasia (A-T) is an infrequent autosomal recessive disorder (OMIM 208900) caused by mutations in the ATM gene encoding the ATM protein, and it was first described in 1957 by Boder et al (1). The ATM gene is located on human chromosome 11q22-q23 and is made up of 66 exons (4 non-coding and 62 coding) spanning $150 \mathrm{~kb}$ of genomic DNA. The ATM gene codes a Ser/Thr kinase (ATM protein) involved in DNA repair that phosphorylates almost two dozen distinct substrates that function in cell signaling to control the cell cycle, repair double-strand DNA breaks, respond to oxidative stress and regulate transcription. The molecular pathogenesis of A-T is abnormal signal transduction of DNA repair and DNA damage (2). The worldwide prevalence of A-T is estimated to be between 1 in 40,000 and 1 in 100,000 live births, and there is no sex predilection (3). More than 1,000 mutations have been reported to date (http://www.hgmd.cf.ac. uk/ac/gene.php?gene=ATM) throughout the gene. Genomic instability affects immunoglobulin coding-related sites and the development of malignant neoplasms. This interference increases the risk of infection and tumorigenesis (4). According to statistics, approximately one-third of A-T patients develop a malignant neoplasm during their lifetime, with a greatly increased incidence of malignant neoplasms in homozygous affected individuals. Heterozygous carriers of the ATM gene are common among the close relatives of patients with A-T. The risk of dying from a malignant neoplasm in A-T heterozygotes was estimated to be over 5 times the respective risk in the general population (5). ATM gene mutations can be identified efficiently and reliably by exon sequencing, which is widely used in the diagnosis of immune deficiency, heredopathia and cancer, providing clinicians with quick guidance on subsequent healthcare management strategies $(6,7)$. In order to enhance the understanding of A-T among clinicians and to promote the early identification and treatment of diseases, we herein report a case of A-T with a hematological malignancy and analyze the 
clinical data and the gene sequence of the whole exome in the patient's pedigree.

\section{Case report}

A 7-year-old male patient was hospitalized at Xiangya Hospital of Central South University (Changsha, China) in September 2017, with complaints of recurrent fever and osteoarticular pain for 20 days that did not improve with antibiotic therapy. The findings on physical examination included superficial lymphadenectasis with movable and non-tender nodes palpated in the neck, axilla and groin (maximum size, $\sim 1.5 \times 1 \mathrm{~cm}$ ), without hepatosplenomegaly. The neurological evaluation revealed instability of gait, abnormal finger-nose test and positive Romberg's sign. Craniocerebral magnetic resonance imaging examination revealed cerebellar atrophy (Fig. 1). The patient's complete blood count (Table I) revealed a differential count of neutrophils $9.0 \times 10^{9} / 1(91.1 \%)$, lymphocytes $0.5 \times 10^{9} / 1(5.4 \%)$ and monocytes $0.3 \times 10^{9} / 1(3.4 \%)$. The patient had microcytic hypochromic anemia (hemoglobin $110 \mathrm{~g} / 1$, mean corpuscular volume $80.6 \mathrm{fl}$, mean corpuscular hemoglobin $26.6 \mathrm{pg}$ and mean corpuscular hemoglobin concentration $330 \mathrm{~g} / \mathrm{l})$. A computed tomography examination of the chest, abdomen and pelvis revealed multiple nodules in the liver and kidney. Bone scans revealed multiple bone metabolic abnormalities throughout the body, including the ribs, vertebrae, tibia and fibula, raising the suspicion of tumor invasion. Immunological testing (Table I) indicated IgA deficiency. Bone marrow cell morphology examination revealed medullary proliferative activity, with primitive and immature lymphocytes accounting for $\sim 78.5 \%$. Flow cytometry analysis of the bone marrow revealed a group of abnormal cells accounting for $22 \%$ of karyocytes, exhibiting weaker expression of CD45 compared with monocytes and slightly more prominent side scatter compared with lymphocytes; these cells were positive for HLA-DR, CD10, CD19, CD20, CD22, CD38, FMC-7 and cCD79a. Immunohistochemical staining revealed the following phenotype: Terminal deoxynucleotidyl transferase ${ }^{-}$, $\mathrm{CD}_{10}^{+}, \mathrm{C}-\mathrm{myc}^{+}(60 \%), \mathrm{CD} 20^{+}, \mathrm{B}-\mathrm{cell}$ lymphoma (Bcl)-2- and Bcl- $6^{+}$. The MIB1 proliferation index was $\sim 100 \%$. The results of fluorescence in situ hybridization were positive for C-MYC gene rearrangement (Fig. 2), suggesting a diagnosis of Burkitt leukemia.

The patient's previous medical history included at least seven upper respiratory infections annually. He was the second child of the family and the perinatal history was unremarkable. The patient's grandfather had been diagnosed with nasopharyngeal carcinoma, but there was no family history of immunodeficiency or neurological disease. The patient started walking at the age of 18 months, with occasional falls. The symptoms of gait instability gradually increased over time, eventually developing into hypotonia and gait dysfunction. The patient was diagnosed with cerebellar ataxia and received rehabilitation training for 6 months; however, his balance did not improve over time.

The patient received sequential chemotherapy in our hospital with the pediatric CCCG-BNHL-2015 regimen for high-risk patients (R4 group) (Table II), which included rituximab, methotrexate (MTX), cytarabine (Ara-C) and cyclophosphamide (CTX), intrathecal (IT) dexamethasone, IT Ara-C and
Table I. Complete blood count and immunological test in the present case.

\begin{tabular}{lcc}
\hline Tests & Value & Normal range \\
\hline White blood cell count $\left(\mathrm{x} 10^{9} / \mathrm{l}\right)$ & 9.9 & $3.5-9.5$ \\
Red blood cell count $\left(\mathrm{x} 10^{12} / \mathrm{l}\right)$ & 4.13 & $4.3-5.8$ \\
Platelet count $\left(\mathrm{x} 10^{9} / \mathrm{l}\right)$ & 581 & $125-350$ \\
Hemoglobin $(\mathrm{g} / \mathrm{l})$ & 110 & $130-175$ \\
Neutrophils $\left(\mathrm{x} 10^{9} / \mathrm{l}\right)$ & 9.0 & $1.8-6.3$ \\
Lymphocytes $\left(\mathrm{x} 10^{9} / \mathrm{l}\right)$ & 0.5 & $1.1-3.2$ \\
Mean corpuscular volume (fl) & 80.6 & $82.0-100.0$ \\
Mean corpuscular hemoglobin (pg) & 26.6 & $27.0-34.0$ \\
Mean corpuscular hemoglobin & 330.0 & $316-354$ \\
concentration (g/l) & & \\
Immunoglobulin G (g/l) & 13.6 & $7.23-16.85$ \\
Immunoglobulin A (mg/l) & $<66.7$ & $690-3,820$ \\
Immunoglobulin M (mg/l) & 2,410 & $630-2,770$ \\
\hline
\end{tabular}

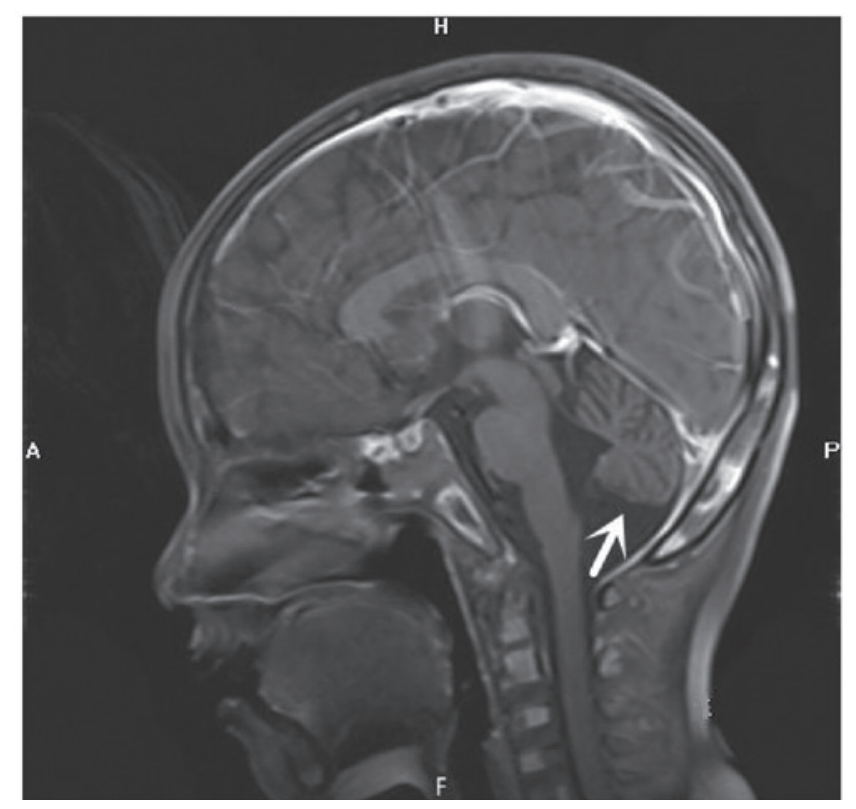

Figure 1. Craniocerebral magnetic resonance imaging examination. Midline sagittal T1-weighted image shows prominent cerebellar folia with mild hypoplasia of the inferior cerebellar vermis and communication of the fourth ventricle with the cisterna magna, consistent with cerebellar atrophy with mild hypoplasia of the inferior cerebellar vermis (arrow).

IT MTX. Recurrent pulmonary infections, oral mucosal ulcers and venous thrombosis have been reported in children during chemotherapy, either during the period of bone marrow suppression or non-bone marrow recovery. Considering the genetic susceptibility and immunological deficiency, whole-exome sequencing was performed, according to the patient's history and clinical manifestations. The results demonstrated that two variants of the ATM gene were associated with A-T, including c.742C > T (p.R248X, 2809; rs730881336) in exon 7 and c.6067-c.6068 ins GAGGGAAGAT (p.G2023Gfs*13) in exon 41. To verify the mutation, after obtaining informed consent, we also sequenced the entire exome of the family 


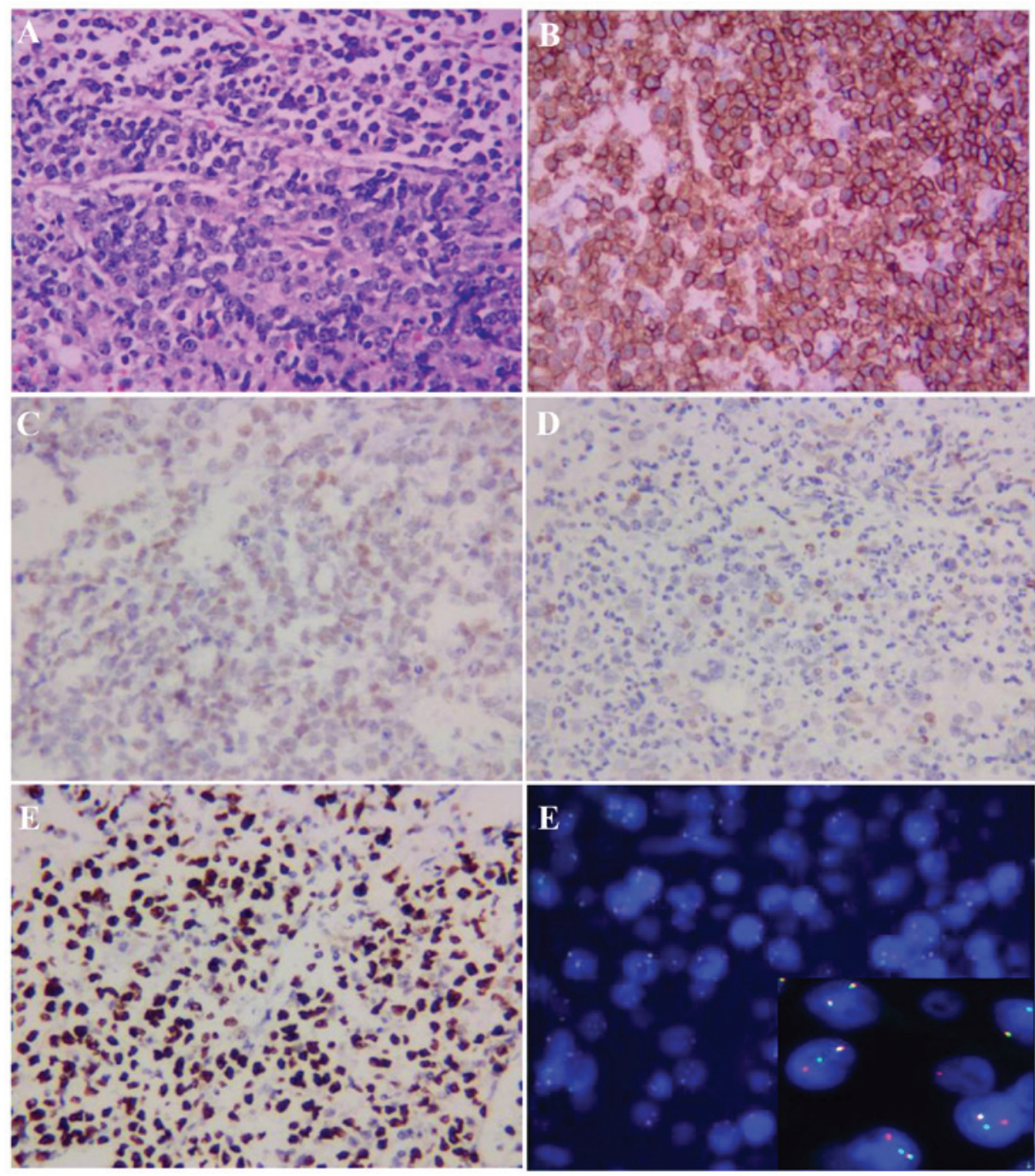

Figure 2. Bone marrow biopsy and molecular examination. Bone marrow biopsy and immunohistochemical staining revealed (magnification, $\mathrm{x} 400)$ : (A) Bone trabeculae with diffuse lymphocytic infiltration (H\&E staining); positive staining for (B) CD20 and (C) C-MYC; negative staining for (D) B-cell lymphoma-2. (E) The Ki-67 proliferation index, as determined by nuclear MIB1 monoclonal antibody staining, was $~ 100 \%$. (F) Fluorescence in situ hybridisation was positive for C-MYC gene rearrangement (insert shows a red, green and yellow signal, with overlapping red and green signals).

members (Table III) and constructed a simplified pedigree chart (Fig. 3). Autosomal complex heterozygosity inheritance was confirmed, as his parents were heterozygous carriers. The patient achieved complete remission after 2 courses of chemotherapy. Unfortunately, his parents refused follow-up treatment and he succumbed to recurrent severe infections 4 months after being diagnosed with Burkitt leukemia.

\section{Discussion}

A-T is a rare autosomal recessive disorder characterized by progressive cerebellar degeneration, telangiectasia, immunodeficiency, recurrent respiratory infections, radiation sensitivity, premature aging and a predisposition to cancer development, particularly of lymphoid origin. Other abnormalities include poor growth, gonadal atrophy, delayed pubertal development and insulin-resistant diabetes. Laboratory abnormalities in A-T commonly manifest as elevated and slowly increasing serum $\alpha$-fetoprotein levels after the age of 2 years, low serum levels of immunoglobulins ( $\operatorname{IgA}$, IgG, IgG subclasses, $\operatorname{IgE}$ ) and lymphopenia (particularly affecting T-lymphocytes) (8).
The majority of the patients manifesting A-T usually succumb to a severe bronchopulmonary infection or malignancy (9). A-T can present with other mobility disorders in addition to ataxia, and a significant proportion of cases do not present with telangiectasia. Thus, Teive et al recommended re-naming this disease ATM syndrome in 2015 (10).

Our patient exhibited early neurological manifestations, such as cerebellar ataxia, but without telangiectasia, and he was not diagnosed with A-T until he developed a hematological malignancy. The diagnosis of A-T can be challenging due to the phenotype that may be incomplete during the disease course. For example, ataxia is usually the earliest clinical manifestation, whereas gait instability progresses slowly and typically becomes obvious after 5 years of age (11). The clinical diagnosis becomes most apparent after the age of 5 years, when ataxia, apraxia and telangiectasia are fully manifested. By contrast, in very young infants, the diagnosis may be more difficult and easily mistaken for mild cerebral palsy, acute infectious or episodic ataxia, or other rare genetic or mitochondrial disorders. Brain magnetic resonance imaging can reveal cerebellar atrophy, but can also be normal in the early stages 
Table II. Pediatric CCCG-BNHL-2015 regimen.

\begin{tabular}{lccc}
\hline & Dose & Administration & Days \\
\hline AA (R4 + rituximab) & & & D1 \\
Cyclophosphamide & $800 \mathrm{mg} / \mathrm{m}^{2}$ & IV over $2 \mathrm{~h}$ & D2,3, 4 \\
Cyclophosphamide & $200 \mathrm{mg} / \mathrm{m}^{2}$ & IV over $1 \mathrm{~h}$ & D1 \\
Vindesine & $3 \mathrm{mg} / \mathrm{m}^{2}(\mathrm{max} 5 \mathrm{mg})$ & IV & D2, D3 \\
Doxorubicin & $20 \mathrm{mg} / \mathrm{m}^{2}$ & IV over $2 \mathrm{~h}$ & D4 (q12 h) \\
Cytarabine & $2,000 \mathrm{mg} / \mathrm{m}^{2}$ & IV over $3 \mathrm{~h}$ & D1-7 \\
Prednisone & $60 \mathrm{mg} / \mathrm{m}^{2}$ & Per os & D1, D8 \\
Triple sheath note (dose by age) & & & D1-5 \\
BB (R4 + rituximab) & & IV over $2 \mathrm{~h}$ & \\
Ifosfamide & $1,200 \mathrm{mg} / \mathrm{m}^{2}$ & & D3-5 \\
& $\mathrm{Mesna} 400 \mathrm{mg} / \mathrm{m}^{2}$, & IV over $2 \mathrm{~h}$ & D1 \\
Etoposide & $0,+4,+8 \mathrm{~h}$ & IV over $24 \mathrm{~h}$ & D1 \\
Methotrexate & $100 \mathrm{mg} / \mathrm{m}^{2}$ & IV & D1-7 \\
Vindesine & $5,000 \mathrm{mg} / \mathrm{m}^{2}$ & Per os & D1, D8 \\
Prednisone & $3 \mathrm{mg} / \mathrm{m}^{2}(\mathrm{max} 5 \mathrm{mg})$ & & \\
Triple sheath note (dose by age) & $60 \mathrm{mg} / \mathrm{m}^{2}$ & & \\
\hline
\end{tabular}

Table III. Results of gene detection in this patient and his family members.

\begin{tabular}{|c|c|c|c|c|c|}
\hline Family member & Exon (ATM gene) & Mutation type & NA changes & AA changes & Prediction \\
\hline \multirow[t]{2}{*}{ Patient } & 7 & Heterozygous & c. $742 \mathrm{C}>\mathrm{T}$ & p.R248X & Harmful \\
\hline & 41 & Heterozygous & $\begin{array}{l}\text { c. } 6067-\text { c. } 6068 \text { ins } \\
\text { GAGGGAAGAT }\end{array}$ & p.G2023Gfs*13 & Harmful \\
\hline \multirow[t]{2}{*}{ Father } & 7 & Heterozygous & c. $742 \mathrm{C}>\mathrm{T}$ & p.R248X & Harmful \\
\hline & 41 & Normal & Normal & Normal & Normal \\
\hline \multirow[t]{2}{*}{ Mother } & 7 & Normal & Normal & Normal & Normal \\
\hline & 41 & Heterozygous & $\begin{array}{l}\text { c.6067-c.6068 ins } \\
\text { GAGGGAAGAT }\end{array}$ & p.G2023Gfs*13 & Harmful \\
\hline \multirow[t]{2}{*}{ Brother } & 7 & Normal & Normal & Normal & Normal \\
\hline & 41 & Heterozygous & $\begin{array}{l}\text { c.6067-c.6068 ins } \\
\text { GAGGGAAGAT }\end{array}$ & p.G2023Gfs*13 & Harmful \\
\hline
\end{tabular}

of the disease (12). Characteristic telangiectasias are rarely observed before the age of 3 years, whereas some patients do not display any signs of capillary dilatation, such as of the eyes and skin, throughout the course of the disease (10). Our patient had IgA deficiency, recurrent infections and chemotherapeutic intolerance. As reported, up to $71 \%$ of patients with A-T who have been investigated had some form of immune deficiency, including low total immunoglobulin levels (IgG, $\operatorname{Ig}$ A or $\operatorname{IgM}$ ), low $\operatorname{IgG} 2$, defective polysaccharide antibody responses and lymphopenia (13). Furthermore, A-T patients with elevated IgM levels constitute a distinct group with a severe disease phenotype and worse prognosis (14); in our patient, IgM was within the normal range. A-T patients usually do not display all the characteristic clinical features, and may exhibit various subtypes and severity of immune deficiency. Patients with gait disorders and recurrent respiratory infections should immedi- ately raise the clinical suspicion of A-T. Early diagnosis can prevent exposure to radiation and enable better management of infections and malignant tumors.

A diagnosis of A-T can be confirmed by the finding of an absence or deficiency of the ATM protein or its kinase activity in cultured cell lines, and/or identification of the pathological mutations in the ATM gene. According to the pedigree chart (Fig. 3), two mutations were found in the ATM gene (located on chromosome 11). The c.742 (exon7) C $>$ T mutation (nonsense mutation, p.R248X, 2809, chr11:108186610-108186611) was detected in patients with capillary dilatation reported in the journal Ann Hum Genet in 2005 by Mitui et al (15), while the ATM mutation c.6067-c.6068 ins GAGGGAAGAT in exon 41 (frameshift mutation, p.G2023Gfs*13, chr11:108115594-108115594) is a novel mutation that was not found in the disease correlation report based on OMIM, 
A

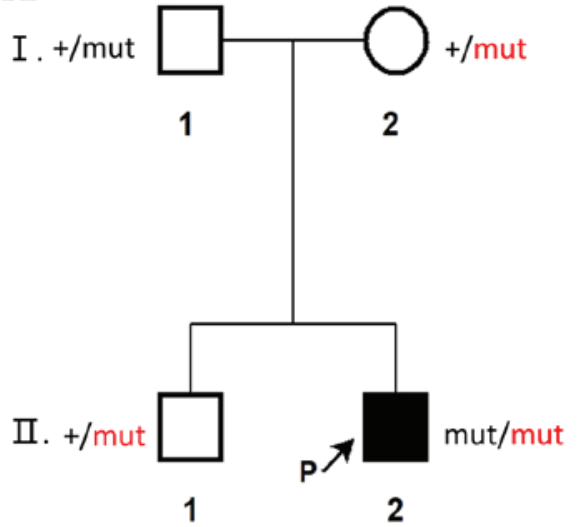

B

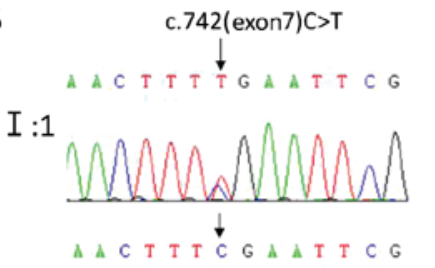

I :2

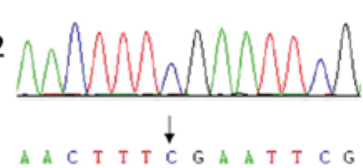

$\Pi: 1$

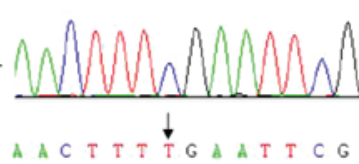

$\Pi: 2$
c.6067(exon41)_c.6068(exon41)insGAGGGAAGAT
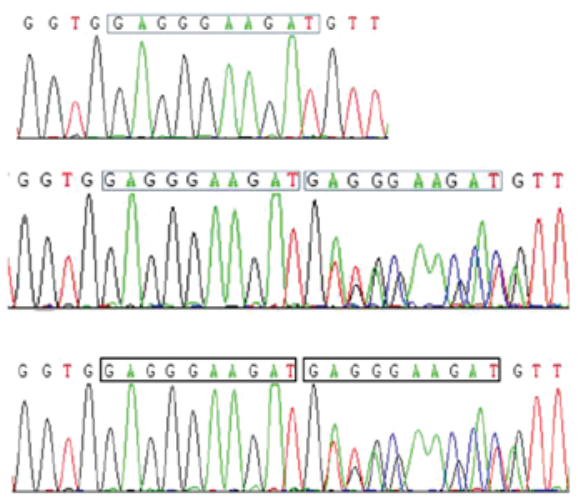

G G T GGAGGGAAGATGAGGG $A \AA G A T G$ T T

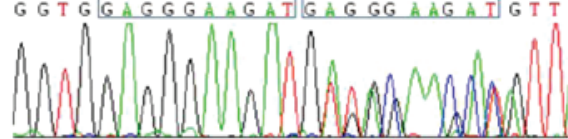

Figure 3. Pedigree chart ATM gene sequencing: (A) Simplified family pedigree. Filled symbols denote patients. The plus (+) denotes the reference sequence, black 'mut' represents c.742(exon7)C $>$ T, and red 'mut' represents c.6067(exon41)_c.6068(exon41) insGAGGGAAGAT. (B) Sanger sequencing showing identified ATM mutations in the family.

HGMD and Clinvar database. This case is very rare, as each family member was a carrier for A-T. Unfortunately, the illnesses in our patient were the result of their double heterozygosity. The difficulties in clinical diagnosis may be overcome by including genetic screening tests in the range of available diagnostic tests, which may also reveal unexpected results. Previous in vitro research has laid the foundation for future mutation targeting therapy in A-T patients, although there is currently no known cure for A-T patients (16).

A-T increases cancer susceptibility [ $25 \%$ lifetime risk of cancers $(17,18)]$, which may be related to the radiosensitivity of A-T resulting in chromosome and chromatid breaks. Tumors are diverse, particularly those of lymphoid origin. The patient's personal and family history is important for identifying and diagnosing tumors. In a retrospective analysis of 279 patients with A-T, 69 had cancer and the majority were hematological cancers, including 38 cases of non-Hodgkin's lymphoma (18). In addition, the families of 17 patients had a history of malignant neoplasms, 11 patients had a family history of recurrent infections and 35 patients had a family history of immunodeficiency in a clinical and laboratory study of $104 \mathrm{~A}-\mathrm{T}$ patients in Iran (19). In addition, it has been found that different mutations express different amounts of ATM proteins with different enzymatic activities. The residual ATM kinase activity is the key to carcinogenesis. Total absence of ATM kinase activity was almost exclusively associated with the development of lymphoid tumors during childhood (17). Therefore, monitoring of ATM kinase activity in A-T patients may contribute to disease prevention and management.

The treatment of A-T is mainly based on medical management of immunodeficiencies and respiratory infections, neurological dysfunction and malignant tumors, supplemented by neurorehabilitation and nutritional counseling. Supportive interventions include education on this disease, genetic counseling, individual and family counseling (20). A-T increases cancer susceptibility. Similarly, aberrancies of the ATM gene are among the most commonly occurring somatic mutations in cancer and have generally been associated with worse prognosis (21). Cancers with deficits in double-stand DNA repair pathways are sensitive to platinum drugs, which are known to act by inducing such double-strand DNA breaks. Conventional chemotherapy regimens for Burkitt leukemia without platinum drugs may not be suitable for such patients. Novel therapies that may improve the response to therapy of patients with ATM-deficient cancers are currently under development, including PARP inhibitors, agents targeting ATR and nucleoside analogues, such as sapacitabine, which induces synthetic lethality (21). The specific problems of managing cancer with A-T are complicated, and treatment should therefore be performed in academic oncology centers following consultation with an A-T specialist. Patients with A-T experience increased toxicity to radio- and chemotherapeutic treatment; therefore, chemotherapy, radiation therapy and radiomimetic drugs should be administered with caution (22). Overall, it is critical that patients and professionals obtain appropriate expert advice and recommendations from multidisciplinary teams on the management of this rare condition, as A-T is a multisystemic disease $(23,24)$.

In conclusion, we herein report a case of A-T with a hematological malignancy and describe two variants of the ATM gene in the patient's family. The gene mutation c.6067-c.6068 ins GAGGGAAGAT in exon 41 (frameshift mutation, p.G2023Gfs"13, chr11:108115594-108115594) detected in this patient is a novel mutation in the ATM gene and, to the best of our knowledge, this is the first report that it may lead to A-T. Detailed medical history, characteristic clinical manifestations and increasingly improved exome sequencing techniques and detection techniques of kinase activity may be beneficial in diagnosing this rare disease, particularly since the initial phenotype of A-T may be incomplete. Management of such diseases should be based on multidisciplinary guidance and other treatment options must be investigated in the future. 


\section{Acknowledgements}

Not applicable.

\section{Funding}

The present study was supported by the National Natural Science Foundation of China (grant nos. 81770178 and 81601528) and the Natural Science Foundation of Hunan Province of China (grant no. 2015J-J6110).

\section{Availability of data and materials}

All data generated or analyzed during this study are included in this published article.

\section{Authors' contributions}

FY: Data collection, biomedical discussion and drafting of the article. LY: Clinical diagnosis and treatment and follow-up of the patient, manuscript elaboration, revision and final approval of the version to be submitted. WC: Histopathological analysis and interpretation of data, revision and final approval of the version to be submitted. MY: Analysis and interpretation of data, revision and final approval of the version to be submitted. MX: Analysis and interpretation of data, revision and final approval of the version to be submitted. All authors have read and approved the final version of the manuscript.

\section{Ethics approval and consent to participate}

Not applicable.

\section{Patient consent for publication}

The legal guardians of both patients provided written informed consent to the publication of the case details.

\section{Competing interests}

The authors declare that they have no competing interests.

\section{References}

1. Boder E and Sedgwick RP: Ataxia-telangiectasia; a familial syndrome of progressive cerebellar ataxia, oculocutaneous telangiectasia and frequent pulmonary infection. Pediatrics 21: 526-554, 1958

2. McKinnon PJ: ATM and the molecular pathogenesis of ataxia telangiectasia. Annu Rev Pathol 7: 303-321, 2012.

3. Gatti RA: Ataxia-telangiectasia. Dermatol Clin 13: 1-6, 1995.

4. Gatti RA: The inherited basis of human radiosensitivity. Acta Oncol 40: 702-711, 2001

5. Swift M, Sholman L, Perry M and Chase C: Malignant neoplasms in the families of patients with ataxia-telangiectasia. Cancer Res 36: 209-215, 1976.

6. Huang Y, Yang L, Wang J, Yang F, Xiao Y, Xia R, Yuan X and Yan M: Twelve novel Atm mutations identified in Chinese ataxia telangiectasia patients. Neuromolecular Med 15: 536-540, 2013.

7. Jeddane L, Ailal F, Dubois-d'Enghien C, Abidi O, Benhsaien I, Kili A, Chaouki S, Kriouile Y, El Hafidi N, Fadil H, et al: Molecular defects in Moroccan patients with ataxia-telangiectasia. Neuromolecular Med 15: 288-294, 2013.
8. Rothblum-Oviatt C, Wright J, Lefton-Greif MA, McGrath-Morrow SA, Crawford TO and Lederman HM: Ataxia telangiectasia: A review. Orphanet J Rare Dis 11: 159, 2016.

9. Su Y and Swift M: Mortality rates among carriers of ataxia-telangiectasia mutant alleles. Ann Intern Med 133: 770-778, 2000.

10. Teive HA, Moro A, Moscovich M, Arruda WO, Munhoz RP, Raskin S and Ashizawa T: Ataxia-telangiectasia-A historical review and a proposal for a new designation: ATM syndrome. J Neurol Sci 355: 3-6, 2015.

11. Chun HH and Gatti RA: Ataxia-telangiectasia, an evolving phenotype. DNA Repair (Amst) 3: 1187-1196, 2004.

12. Gatti RA, Becker-Catania S, Chun HH, Sun X, Mitui M, Lai CH, Khanlou N, Babaei M, Cheng R, Clark C, et al: The pathogenesis of ataxia-telangiectasia. Learning from a Rosetta Stone. Clin Rev Allergy Immunol 20: 87-108, 2001.

13. Nowak-Wegrzyn A, Crawford TO, Winkelstein JA, Carson KA and Lederman HM: Immunodeficiency and infections in ataxia-telangiectasia. J Pediatr 144: 505-511, 2004.

14. Krauthammer A, Lahad A, Goldberg L, Sarouk I, Weiss B, Somech R, Soudack M and Pessach IM: Elevated IgM levels as a marker for a unique phenotype in patients with Ataxia telangiectasia. BMC Pediatr 18: 185, 2018.

15. Mitui M, Bernatowska E, Pietrucha B, Piotrowska-Jastrzebska J, Eng L, Nahas S, Teraoka S, Sholty G, Purayidom A, Concannon P and Gatti RA: ATM gene founder haplotypes and associated mutations in Polish families with ataxia-telangiectasia. Ann Hum Genet 69: 657-664, 2005.

16. Nakamura K, Du L, Tunuguntla R, Fike F, Cavalieri S, Morio T, Mizutani S, Brusco A and Gatti RA: Functional characterization and targeted correction of ATM mutations identified in Japanese patients with ataxia-telangiectasia. Hum Mutat 33: 198-208, 2012.

17. Reiman A, Srinivasan V, Barone G, Last JI, Wootton LL, Davies EG, Verhagen MM, Willemsen MA, Weemaes CM, Byrd PJ, et al: Lymphoid tumours and breast cancer in ataxia telangiectasia; substantial protective effect of residual ATM kinase activity against childhood tumours. Br J Cancer 105: 586-591, 2011.

18. Suarez F, Mahlaoui N, Canioni D, Andriamanga C, Dubois d'Enghien C, Brousse N, Jais JP, Fischer A, Hermine O and Stoppa-Lyonnet D: Incidence, presentation, and prognosis of malignancies in ataxia-telangiectasia: A report from the French national registry of primary immune deficiencies. J Clin Oncol 33: 202-208, 2015.

19. Moin M, Aghamohammadi A, Kouhi A, Tavassoli S, Rezaei N, Ghaffari SR, Gharagozlou M, Movahedi M, Purpak Z, Mirsaeid Ghazi B, et al: Ataxia-telangiectasia in Iran: Clinical and laboratory features of 104 patients. Pediatr Neurol 37: 21-28, 2007.

20. Perlman S, Becker-Catania S and Gatti RA: Ataxia-telangiectasia: Diagnosis and treatment. Semin Pediatr Neurol 10: 173-182, 2003.

21. Choi M, Kipps T and Kurzrock R: ATM mutations in cancer: Therapeutic implications. Mol Cancer Ther 15: 1781-1791, 2016.

22. Schoenaker MH, Suarez F, Szczepanski T, Mahlaoui N and Loeffen JL: Treatment of acute leukemia in children with ataxia telangiectasia (A-T). Eur J Med Genet 59: 641-646, 2016.

23. van Os NJH, Haaxma CA, van der Flier M, Merkus PJFM, van Deuren M, de Groot IJM, Loeffen J, van de Warrenburg BPC and Willemsen MAAP; A-T Study Group: Ataxia-telangiectasia: Recommendations for multidisciplinary treatment. Dev Med Child Neurol 59: 680-689, 2017.

24. Bhatt JM, Bush A, van Gerven M, Nissenkorn A, Renke M, Yarlett L, Taylor M, Tonia T, Warris A, Zielen S, et al: ERS statement on the multidisciplinary respiratory management of ataxia telangiectasia. Eur Respir Rev 24: 565-581, 2015.

This work is licensed under a Creative Commons Attribution-NonCommercial-NoDerivatives 4.0 International (CC BY-NC-ND 4.0) License. 